\title{
Performance Evaluation of a QoS-aware Error Control Scheme for Multiple- WBAN Environment
}

\author{
Kento Takabayashi*a), Non-Member, \\ Tuomas Paso**, Non-Member, \\ Heikki Karvonen**, Non-Member, \\ Chika Sugimoto*, Non-Member, Ryuji Kohno*, Member
}

(Manuscript received Jan. 00, 20XX, revised May 00, 20XX)

\begin{abstract}
This paper presents a comprehensive performance evaluation of an error control scheme using decomposable codes while satisfying Quality of Service (QoS) requirements in a multiple-Wireless Body Area Network (WBAN) environment. Our QoS control scheme comprised a multiplexing layer and decomposable error control coding used to adapt the communication parameters and satisfy the different performance requirements of different types of WBAN data traffic. Using mathematical analysis and simulations, it has been shown in previous studies that this method has a better residual bit error ratio (RBER) and throughput performance than that of an IEEE 802.15.6-based solution. The energy efficiency of the proposed method was recently evaluated for an additive white Gaussian noise case. In this work, this performance evaluation was complemented by simulating the RBER performance under a defined worst-case scenario. The energy efficiency was compared with that of the IEEE 802.15.6 solution using a realistic WBAN channel model and accounting for the interference from neighboring WBANs. Results from the previous studies and the new results are given to provide a complete description of the performance of our method. The results demonstrate that an error control scheme based on decomposable codes satisfies the QoS requirements of different data types and provides a more flexible and energy efficient QoS control than that of the IEEE 802.15.6 solution. The scheme was also shown to guarantee communication performance in the worst-case scenario, whereas the IEEE 802.15.6 solution failed to do so.
\end{abstract}

\section{Keywords : Wearable sensor, Wireless Body Area Network, QoS control, Decomposable codes, Energy efficiency, Multiple-WBAN interference}

\section{Introduction}

The ratio between the old and young in the global population is more rapidly increasing than that in the past. This is raising concerns regarding the increasing cost of medical care. It is also thought that people will become increasingly health conscious in the future. Meanwhile, portable devices such as smart phones, tablets, and wearable devices have become widespread. Health monitoring systems employing wearable vital sensors and using wireless communication (also known as m-health or m-IoT) have received significant attention in recent years [1]-[10]. It is expected that m-IoT will allow continuous monitoring of the user's physical condition and help preempt serious illnesses. In the field of m-IoT systems, the wireless body area network (WBAN) is a key technology [11]-[14]. Extensive work on the standardization of such systems has been carried out [9]-[10].

In a WBAN system, a wearable vital sensor node can use a range of sensor types, all of which have different data rates. The allowable communication error ratio or delay depends on the application used. IEEE Std. 802.15.6 defines eight levels of user

a) Correspondence to: Kento Takabayashi. E-mail: takabayashi-kentoxp@ynu.jp

* Graduate School of Engineering, Yokohama National University, 79-5 Tokiwadai, Hodogaya-Ku, 240-8501 Yokohama, Japan

** Centre for Wireless Communications (CWC), University of Oulu, P.O.Box 4500, 90014 Oulu, Finland

$* * *$ Graduate School of Information Sciences, Hiroshima City University, 3-4-1, Ozuka-Higashi, Asa-Minami-Ku, 731-3194 Hiroshima, Japan priorities, as shown in Table 1 [9]. The Quality of Service (QoS) control must ensure that different types of data can be communicated as efficiently as possible at a satisfactory quality level. The optimal QoS control of input data is therefore an important factor in sensor data transmission [11]-[14].

To address this requirement, we have proposed an optimal QoS control scheme employing a multiplexing layer for priority scheduling and a decomposable error control coding scheme that can adapt to varying channel conditions [11]. The target WBAN is a wearable device comprising multiple sensors whose output data is transmitted using a common medium access control (MAC) and PHY layer [11]. Simulations were conducted to evaluate the performance of the proposed system and to compare its performance with that of a system based on IEEE 802.15.6 [11]. We earlier presented a theoretical analysis of the proposed method in [12], and derived a lower bound on throughput and an upper bound on the residual bit error rate for both the proposed system and the standard system under the additive white Gaussian noise (AWGN) channel and the Rayleigh fading channel. In the same study, we investigated the QoS parameters to allow optimization of our system [12]. However, the study did not take account of interference from other WBANs, and the worst-case performance has not been evaluated in previous studies [11]-[12].

In [13], the residual bit error ratio (RBER) and throughput performances of the proposed scheme was evaluated in a multiple WBAN environment, representing a more realistic situation than those in [11]-[12]. A further study investigated the energy efficiency of the proposed scheme in the AWGN channel using computer 
simulations [14]. Energy efficiency is an important factor because WBAN nodes are small and their battery capacity is limited. It is therefore crucial to evaluate the energy efficiency of the proposed decomposable error control codes using a more realistic channel model.

To complete the performance evaluation of the proposed scheme, this study quantitatively evaluated the RBER and energy efficiency of our proposed system under a WBAN channel model not only in a general case, but also in the worst-case scenario. High levels of reliability are required in medical applications, especially when channel conditions are poor. The difference in each performance metric between the general and worst cases provided a clear performance comparison. In previous work, the performance evaluation was conducted for differential binary phase shift keying (DBPSK) modulation. In this work, differential quadrature phase shift keying (DQPSK), as defined in IEEE 802.15.6, was also investigated. Simulation results showed that the proposed scheme was able to achieve a strong performance and to guarantee a satisfactory QoS in both scenarios, while the IEEE Std. 802.15.6 solution failed to meet the QoS requirements, especially in the worst case scenario.

The rest of this paper is organized as follows. In Section 2, we introduce the 15.6 Std. In Section 3, the system model of our scheme is given. Section 4 describes our decomposable error correction code and the working process of our proposed system. Section 5 sets out the energy efficiency model. The results of our performance evaluations using computer simulation are given in Section 6. Finally, we present our conclusions in Section 7.

\section{IEEE Std. 802.15.6}

In this section, we describe the relevant parts of IEEE 802.15.6. A more detailed account of IEEE 802.15.6 is presented in [9].

2.1 Modes of operation The 15.6 Std. defines three PHY layers: narrowband (NB), ultra-wideband (UWB), and human body communications (HBC). In this study, we focused on an impulse radio ultra-wideband PHY layer (IR-UWB-PHY) which offers high data rate transmission, low energy consumption, and good coexistence with other wireless communication systems. In the UWB-PHY of IEEE 802.15.6, there are two modes of operation: default mode and high QoS mode. The default mode can be used in both medical and non-medical applications. The high QoS mode is for use in medical applications which have a user priority of six.

\subsection{UWB PHY frame format The UWB PHY frame} format is formed by the synchronization header (SHR), the physical layer header (PHR), and the physical layer service data unit

(PSDU), respectively, as shown in Figure 1 [9]. The PSDU contains the MAC protocol data unit (MPDU) and the $\mathrm{BCH}$ parity bits in default mode, while the PSDU contains either the MPDU or $\mathrm{BCH}$ parity bits in high QoS mode. The PSDU can therefore be regarded as the payload. The information contained by the PHR includes the data rate of the PSDU and the length of the MAC frame body, and the SHR contains the preamble used for timing synchronization, packet detection and other purposes, and the startof-frame delimiter (SFD) for frame synchronization. In this research we focused mainly on the performance of the payload (PSDU).

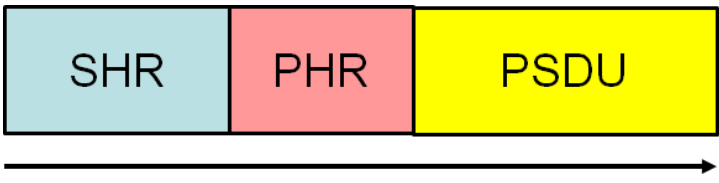

\section{Transmit order}

Fig. 1. UWB PHY frame structure [9].

2.3 Error control scheme for the UWB-PHY In these modes of operation, the channel codes applied to the PSDU are $(63,51) \mathrm{BCH}$ code in the default mode defined in UWB-PHY and NB-PHY and $(126,63)$ shortened BCH code in the high QoS mode defined in UWB-PHY. In the high QoS mode, the $(126,63)$ shortened $\mathrm{BCH}$ encoder uses the hybrid ARQ mechanism and the $(126,63)$ shortened $\mathrm{BCH}$ code is derived from the mother code $(127$, 64) BCH. The type II hybrid ARQ scheme is used in the high QoS mode. The process can be summarized as follows:

(1) A packet D is encoded with the $(126,63)$ shortened $\mathrm{BCH}$ code. The output of the encoder consists of parity bits $\mathrm{P}$ and systematic bits D of the same length. Both D and $\mathrm{P}$ are stored at the transmitter, but only $\mathrm{D}$ is transmitted. If such a transmitted packet is detected in error by the CRC-16-CCITT code, $\mathrm{D}$ is not discarded but is stored at the receiver.

(2) Upon No acknowledgment (N-ACK) at the transmitter, P is transmitted and the information bits are recovered by $(126,63)$ $\mathrm{BCH}$ decoding with the stored $\mathrm{D}$ and received $\mathrm{P}$.

(3) If the $(126,63) \mathrm{BCH}$ decoding fails, the $\mathrm{D}$ stored at the receiver is discarded and $\mathrm{P}$ is stored instead. Upon N-ACK at the transmitter, the previously stored D at the transmitter is sent again.

(4) If such a retransmitted D is detected in error, $(126,63) \mathrm{BCH}$ decoding is applied to the received $\mathrm{D}$ and the stored P. If (126, 63) $\mathrm{BCH}$ decoding fails and the maximum number of retransmissions has not been reached, the stored $\mathrm{P}$ is discarded, the received $\mathrm{D}$ is stored, and $\mathrm{P}$ is retransmitted upon $\mathrm{N}-\mathrm{ACK}$ at the transmitter.

(5) This process is repeated until D is successfully received or the maximum number of retransmissions is reached. The maximum number of retransmissions for the hybrid ARQ is set to four.

IEEE 802.15.6 defines such an error control method. However, in the default mode, the error correction capacity is increased by repeated retransmission, as only a single $\mathrm{BCH}$ code is used. This limits the error correcting capacity of the high QoS mode because both the maximum number of retransmissions and the coding rate of the error correcting code are fixed. The error control scheme of the standard cannot therefore meet the various QoS requirements for WBAN data.

\section{System model}

We assumed that the sensor node comprised multiple sensors transmitting different types of data to the hub, as shown in Figure 2. In this study, two data types (Data A and Data B) with different applicable QoS standards were considered. Low RBER is required for Data A, while low redundancy is needed for Data B, to maximize energy efficiency and reduce latency. As the first reason for selecting those QoSs, we particularly focused on an error controlling scheme utilizing Hybrid ARQ in the paper, and then RBER and the energy efficiency are very important parameters for 
an evaluation of such a scheme. Secondly, those two parameters are related to a trade-off. So, we also aimed to show the relationship in some evaluations. Data A was assumed to be a physiological parameter with a low data rate, for example blood pressure, $\mathrm{SpO}$, or temperature, and Data B to be a waveform such as an ECG output. The transmission order and error control processes of different types of data packet depend on the QoS level required. Table 1 summarizes the characteristics of the different data types. The required data rates were taken from [15]. RBER is the ratio of bit errors when the maximum number of retransmissions has been reached.

In addition, each WBAN operated independently and interfered with the others, as shown in Figure 3.

The transmitter consisted of a multiplexing module, a MAC module, and a PHY module [11]-[14]. The MUX (multiplexer) controller in the multiplexing module controlled the multiple QoS levels, based on these priorities. First, data was added to the user priorities. The header, including the user priority information (e.g., the latency, rate of error control coding, number of repetitions in ARQ, etc.) was then added to the user priorities in the multiplexing layer. Next, the multiplexer controller in the multiplexing layer provided instructions to each data set based on predefined parameters. In response to the QoS control signal, the multiplexing layer performed error and delay control. Finally, data with different user priorities were multiplexed and sent to the MAC layer [11][12].

In the IEEE 802.15.6 definitions, data can be modulated at the UWB PHY layer by using either on-off keying (OOK) in the default mode and optionally in the high QoS mode, or differentially encoded phase-shift keying (DPSK) in the high QoS mode and optionally in the default mode [9]. In this research, DBPSK and DQPSK were used because DPSK modulation has higher robustness against errors than $\mathrm{OOK}$, and this modulation scheme can also be more easily simulated. The DPSK transmitting symbols are given by following equation:

$$
c_{m}=c_{m-1} \exp \left(j \varphi_{m}\right)
$$

where $c_{m}$ is the $\mathrm{m}^{\text {th }}$ encoded DBPSK or DQPSK symbol, $\mathrm{m}=$ $(0,1, \ldots, N), N$ gives the number of symbols, $c_{-1}=1$, and $\varphi_{0}$ is an arbitrary phase. The symbol $c_{m}$ carries either one (DBPSK) or two (DQPSK) bits of information. The mapping of information bits onto $\varphi_{m}$ is given in Tables 2 and 3 .

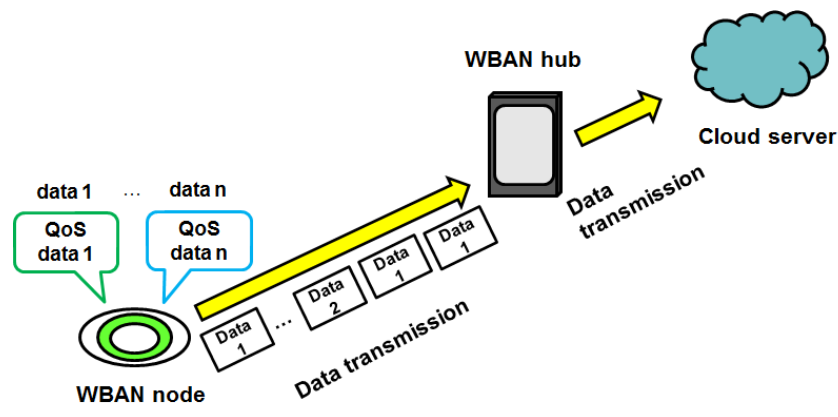

Fig. 2. System concept for a single WBAN [11]-[14].

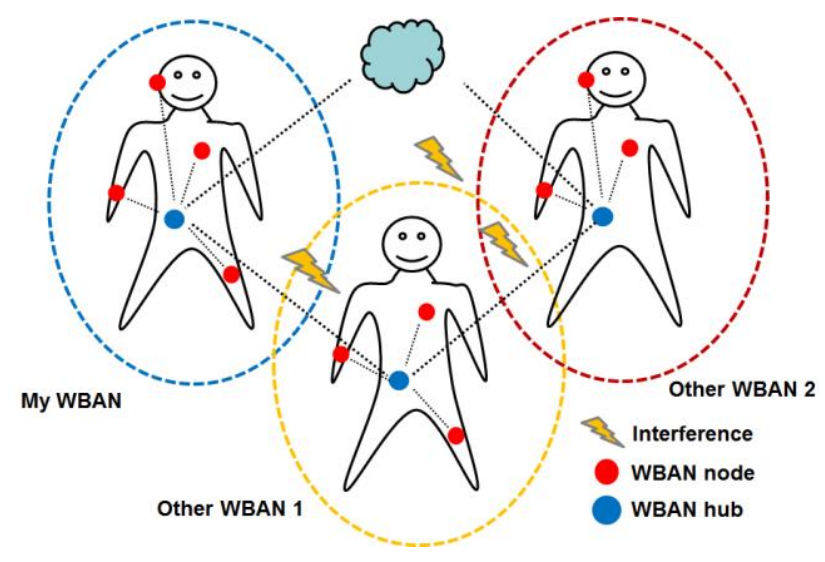

Fig. 3. Multiple WBAN environment [13]-[14].

Table 1. Characteristics of different data types.

\begin{tabular}{|l|l|l|}
\hline Data Types & Data A & Data B \\
\hline User Priority & 5 & 6 \\
\hline RBER & $\begin{array}{l}\text { Restricted } \\
<10^{-6}\end{array}$ & $\begin{array}{l}\text { Allowable } \\
<10^{-2}\end{array}$ \\
\hline $\begin{array}{l}\text { The maximum } \\
\text { retransmissions, } q\end{array}$ & 10 & 4 \\
\hline Required data rate & $160 \mathrm{bps}$ & $2.4 \mathrm{kbps}$ \\
\hline
\end{tabular}

Table 2. Mapping of information bits onto $\varphi_{m}$ for DBPSK.

\begin{tabular}{|l|l|}
\hline$g_{m}$ & $\varphi_{m}$ \\
\hline 0 & 0 \\
\hline 1 & $\pi$ \\
\hline
\end{tabular}

Table 3. Mapping of information bits onto $\varphi_{m}$ for DBPSK.

\begin{tabular}{|l|l|l|}
\hline$g_{2 m}$ & $g_{2 m+1}$ & $\varphi_{m}$ \\
\hline 1 & 1 & $\pi / 2$ \\
\hline 0 & 1 & $\pi$ \\
\hline 0 & 0 & $-\pi / 2$ \\
\hline 1 & 0 & 0 \\
\hline
\end{tabular}

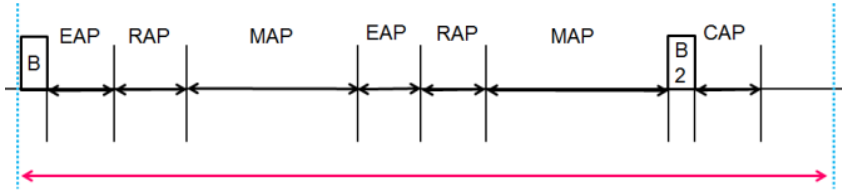

Beacon period (Superframe)

Fig. 4. Beacon mode with superframes (mode I) [9].

To increase robustness against multipath fading and multi-user interference, a direct spread spectrum (DSSS) technique can be used for the DPSK modulated data. The effectiveness of the DSSS technique in a multiple WBAN environment in a hospital was demonstrated in [16].

In the MAC layer, this study assumed a beacon mode with super frames, following [9]. In this mode, a hub sets the access phase, such as an exclusive access phase (EAP), random access phase (RAP), managed access phase (MAP), or contention access phase (CAP). This is illustrated in Figure 4. A hub may set the length of 
any of these access phases to zero. In this study, only MAP was used to reflect the requirement for medical data to be transmitted more reliably than non-medical data. A hub and nodes may send data by using scheduled allocations, preventing collisions from occurring in the same WBAN. If a random access protocol is also used, the required delay, energy consumption, or number of retransmissions will increase because of packet collisions. These factors cannot then be guaranteed, and their dependability is reduced. Some ACK policies, including I-ACK and G-ACK and so on, are defined in IEEE 802.15.6 [9]. In this research we assumed that I-ACK was sent immediately if the packet was sent successfully, while N-ACK was sent if the packet transmission failed.

\section{Decomposable error control coding}

In our previous studies, a decomposable error control code was used to optimize the QoS levels of different data types [11]-[14]. As an example of a decomposable code, a punctured convolutional code with a constraint length $K=3$ and coding rates of 8/9 1/2 was used as a reference [11]-[12]. In [13]-[14], the coding rate was extended to $1 / 16$.

The punctured convolutional code was generated from a convolutional code whose generator polynomial was $[7,5]$ and code rate was $r=1 / 2$. The punctured matrix of $r=k_{0} / v_{0}=8 / 9$ is shown in Figure 5.

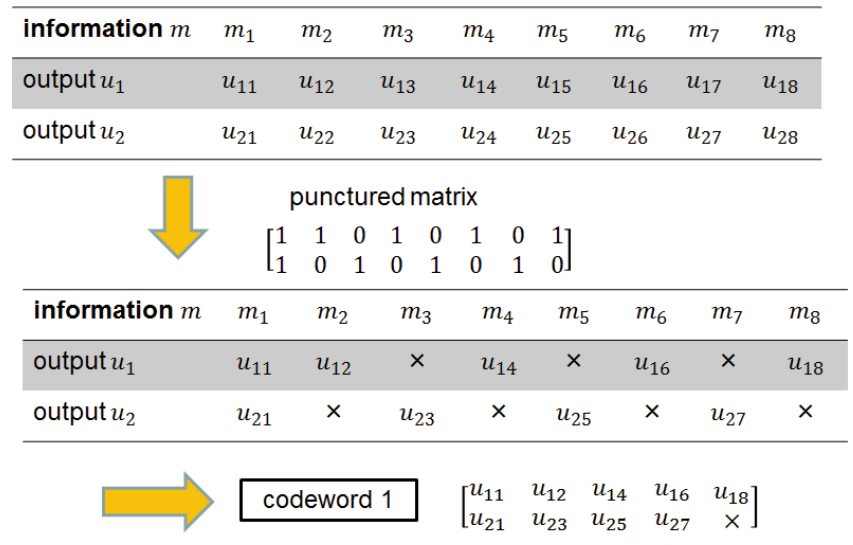

\begin{tabular}{lcccccccc}
\hline information $m$ & $m_{1}$ & $m_{2}$ & $m_{3}$ & $m_{4}$ & $m_{5}$ & $m_{6}$ & $m_{7}$ & $m_{8}$ \\
\hline output $u_{1}$ & $u_{11}$ & $\times$ & $u_{13}$ & $\times$ & $u_{15}$ & $\times$ & $u_{17}$ & $\times$ \\
output $u_{2}$ & $u_{21}$ & $u_{22}$ & $\times$ & $u_{24}$ & $\times$ & $u_{24}$ & $\times$ & $u_{28}$ \\
\hline
\end{tabular}

$$
\text { codeword 1' }\left[\begin{array}{llllc}
u_{11} & u_{13} & u_{15} & u_{17} & \times \\
u_{21} & u_{22} & u_{24} & u_{26} & u_{28}
\end{array}\right]
$$

Fig. 5. Punctured convolutional code $r=8 / 9$ [12]-[14].
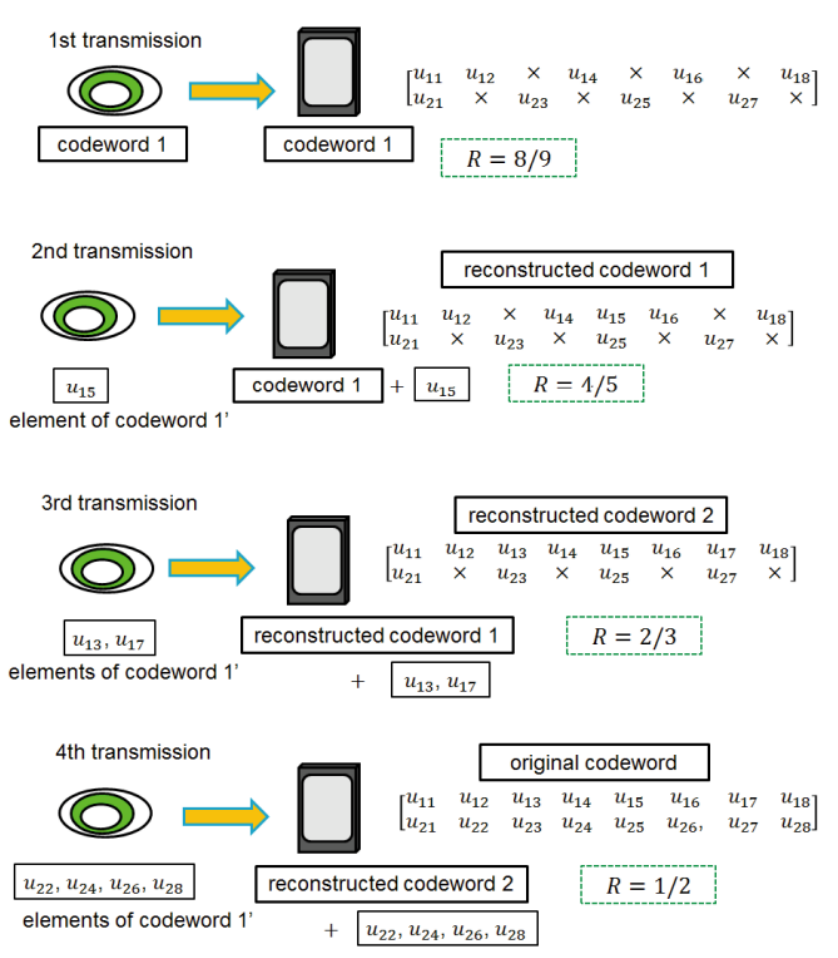

Fig. 6. Method of reconstructing decomposable codes $r=8 / 9$ $1 / 2$ [12]-[14].
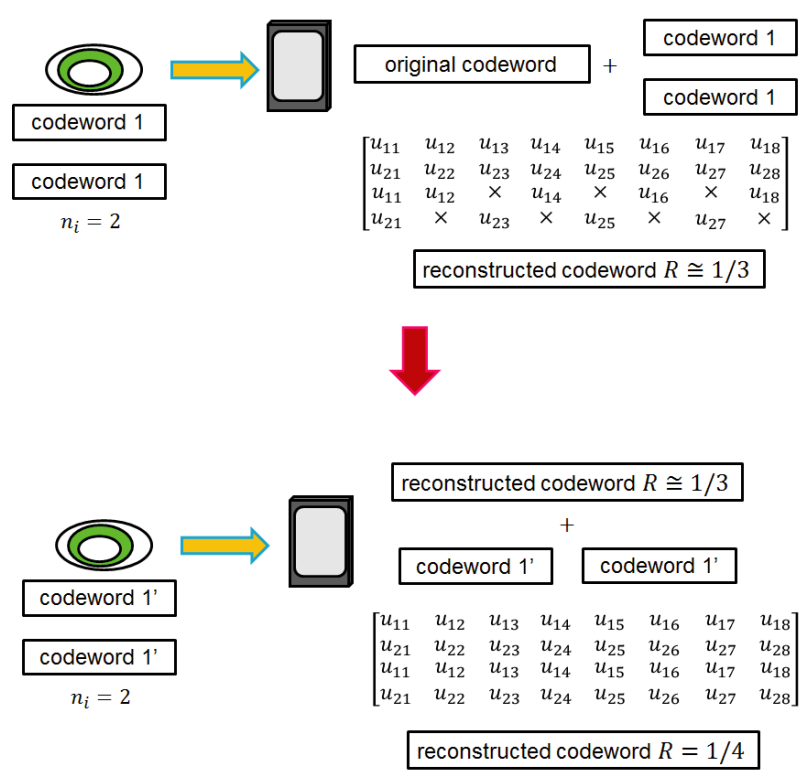

Fig. 7. Example of method of reconstructing decomposable codes $r=1 / 2 \sim[13]-[14]$

Table 4. The number of data copies in Weldon's ARQ,

$n_{i}$ and the number of transmitted encoded bits $m_{i}$

\begin{tabular}{|l|c|c|c|c|c|c|c|c|c|c|c|}
\hline$i$ & 0 & 1 & 2 & 3 & 4 & 5 & 6 & 7 & 8 & 9 & 10 \\
\hline Data A, $n_{i}$ & 1 & 4 & 4 & 5 & 5 & 6 & 6 & 8 & 8 & 8 & 8 \\
\hline Data B, $n_{i}$ & 1 & 1 & 2 & 3 & 4 & & & & & & \\
\hline$m_{i}$ & 9 & 1 & 2 & 4 & 9 & 9 & 9 & 9 & 9 & 9 & 9 \\
\hline
\end{tabular}




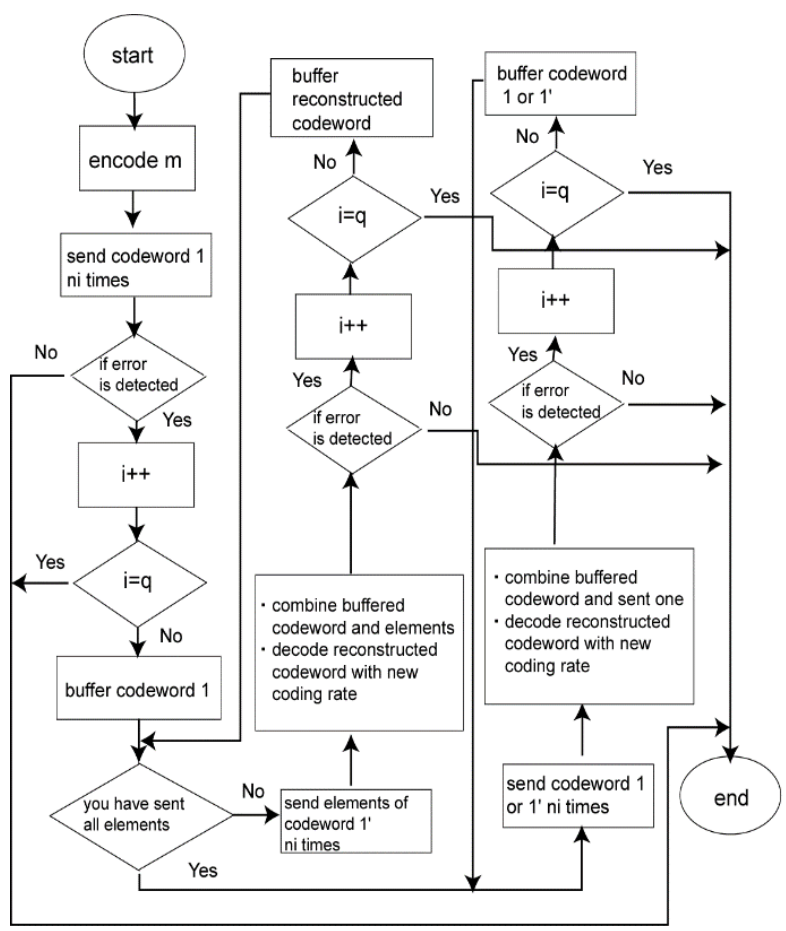

Fig. 8. Flowchart of the proposed ARQ protocol [13]-[14].

The two patterns of the $r=8 / 9$ punctured codes (code words 1 and 1') were generated using this punctured matrix. At the first transmission time, the code word 1 was sent. To increment the code rate of the punctured code, elements of the code word 1' were then sent after the first transmission, as shown in Figure 6. After all elements for reconstructing the original convolutional code had been sent, the code words 1 and 1' were alternately transmitted. An example is given in Figure 7. Finally, the receiver reconstructed and decoded any low-rate decomposable code by changing the number of data copies using Weldon's ARQ protocol [17].

In the proposed scheme, retransmission was performed as follows. First, the information $m$ was encoded via the punctured convolutional code, and code word 1 from Fig. 5 was transmitted. If errors were detected, the receiver stored the transmitted code word 1 and the transmitter re-sent the subcode word of code word $1^{\prime} n_{i}$ times if $1 \leq i \leq 3$. At the receiver, the received sub code word and stored code word were combined (Fig. 6) and the reconstructed code word was decoded. After the third retransmission, code word 1 was sent $n_{4}$ times and combined with a buffered codeword. If errors were detected, the $n_{4}$ codeword 1 was buffered in the receiver, and codeword 1' was transmitted $n_{5}$ times and combined with a stored code word. After that, code words 1 and 1 ' were sent alternately $n_{i}$ times and stored. The transmitter repeated these operations until the data had been correctly received or the predefined maximum number of retransmissions had been reached.

In this research, the parameters of Weldon's ARQ protocol were set as shown in Table 4. A buffered existing code word was updated to become a new transmitted code word. The error correction ability increased as the coding rate decreased, in the order of 8/9 1/16. It is also possible to combine this coding scheme with link adaptation for channel condition [18]. However, this approach was not used, as the parameters were preset to allow our system to adapt to each QoS.

Figure 8 shows a flowchart of the protocol of the proposed scheme. The operation was continued until no further errors were detected or until the maximum number of retransmissions $q$ was reached.

\section{Energy efficiency model}

We based our energy consumption model on those in [14] and [19]. The energy consumption for the communication link was calculated as follows:

$$
\begin{gathered}
E_{\text {link }}=\left(T_{T O T}+N_{t x} T_{A C K}\right)\left(P_{t x, R F}+P_{t x, \text { circ }}\right. \\
\left.+P_{r x}\right)+N_{t x}\left(\varepsilon_{\text {enc }}+\varepsilon_{\text {dec }}\right) \\
T_{T O T}=\sum_{i=1}^{N_{t x}} \frac{L_{\text {packet }, i}}{R} \\
L_{\text {packet }, i}=L_{P H R}+L_{S H R}+L_{P S D U, i} \\
L_{P S D U, i}=\frac{n_{i} m_{i}}{k_{0}} L_{\text {info }} \\
T_{A C K}=\frac{L_{A C K}}{R}
\end{gathered}
$$

where $L_{A C K}$ is the acknowledgement (ACK) packet length, $R$ is the uncoded data rate, $T_{A C K}$ is the duration of an ACK packet, $L_{P H R}$ is the length of a physical layer header (PHR), $L_{S H R}$ is the length of a synchronization header (SHR), $L_{P S D U, i}$ is the length of a physical layer service data unit (PSDU) for the $i$ th transmission, $L_{\text {packet }, i}$ is the length of the $i$ th transmission, $N_{t x}$ is the number of transmissions, $T_{T O T}$ is the duration of the $N_{t x}$ transmissions, $P_{t x, R F}$ is the transmitter RF power consumption, $P_{t x, c i r c}$ is the transmitter circuitry power consumption, $P_{r x}$ is the receiver power consumption, $\varepsilon_{e n c}$ and $\varepsilon_{\text {dec }}$ are the encoding and decoding energies, $k_{0}$ is the number of uncoded bits, $m_{i}$ is the number of transmitted encoded bits, $n_{i}$ is the number of copy blocks of Weldon's ARQ at the $i$ th transmission and $L_{\text {info }}$ is the number of information bits in a code word. The PSDU length is changed in every retransmission following the used decomposable ECC method.

The encoding and decoding energy consumption for the decomposable coding were calculated based on the model introduced in [20] as

$$
\begin{aligned}
& \varepsilon_{\text {enc }}=\frac{P_{\text {convenc }}}{R} L_{\text {info }}^{2} \\
& \varepsilon_{\text {dec }}=\frac{P_{\text {vitdec }}}{R} L_{\text {info }}^{2}
\end{aligned}
$$

Here, $P_{\text {convenc }}[\mathrm{nW} / \mathrm{bit}]$ is the encoding power of a convolutional code and $P_{\text {vitdec }}[\mathrm{nW} / \mathrm{bit}]$ is the decoding power of Viterbi decoding. The values of $P_{\text {convenc }}$ and $P_{\text {vitdec }}$ were taken from [20]. Finally, the energy efficiency was defined as 


$$
\eta \equiv \frac{\lambda}{E_{\text {link }}}
$$

where $\lambda$ is the number of successfully received information bits after $N_{T x}$ transmission trials, excluding redundancy or header bits. Hence, $\lambda$ was expressed as follows:

$$
\lambda=\left\{\begin{array}{cc}
L_{\text {info }} & \text { (If sent successfully) } \\
0 \text { (If not) }
\end{array}\right.
$$

\section{Performance evaluations}

\subsection{Simulation conditions}

In this section, the simulation model developed for the performance evaluation of the proposed scheme is described.

Two types of situation were considered, as shown in Figure 9. Here,

$d_{j}$ means the distance from the $j$ th other WBAN of an objective

WBAN. In Case 1, a general case, other WBANs followed a uniform distribution within three meters of the objective WBAN. A path loss of transmission power from the other WBANs was treated as a free space propagation loss. IEEE802.15.6 CM4 [21] was applied to the channel model from the other WBANs. IEEE802.15.6 CM4 defines a channel model between a WBAN node or hub and an external device. In Case 2, the other WBANs were placed more closely to the objective WBAN. This case applied IEEE802.15.6 CM3 [21], which is a channel model for a wearable WBAN under interference from other WBANs. In this scenario, the other WBANs were not regarded as external devices but as an asynchronous wearable WBAN node. This constituted the worst-

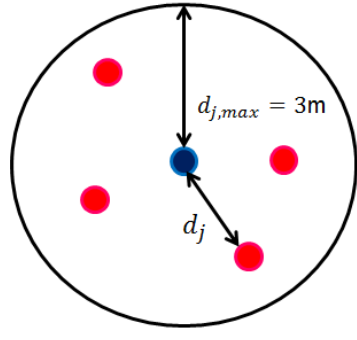

(1) case 1 - general case

Objective WBAN

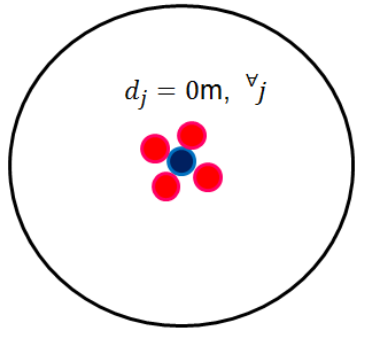

(2) case 2 - worst case

Other WBAN
Fig. 9. Two types of cases in computer simulations. (1) and (2) present case 1 (general case) and case 2 (worst case) [13],

\begin{tabular}{|c|c|}
\hline Parameter & Detail \\
\hline Channel model & IEEE802.15.6 CM3, CM4 \\
\hline Bandwidth & $499.2 \mathrm{MHz}$ \\
\hline Central frequency & $3.99 \mathrm{GHz}$ \\
\hline Modulation & DBPSK, DQPSK \\
\hline FEC & $\begin{array}{l}\mathrm{R}=8 / 9 \sim 1 / 16, K=3 \\
\text { convolutional codes }\end{array}$ \\
\hline Decoding & $\begin{array}{l}\text { Soft decision } \\
\text { Viterbi decoding }\end{array}$ \\
\hline ARQ protocol & Weldon's ARQ \\
\hline Spreading sequence & Gold sequence \\
\hline Spreading Factor, $N_{s}$ & 7,15 \\
\hline Pulse shape & Gaussian monopulse \\
\hline The number of other WBANs & $0 \sim 14$ \\
\hline Distance from other WBANs & $3 \mathrm{~m}$ \\
\hline$L_{\text {info }}$ & 306 bits \\
\hline$L_{A C K}$ & 7 bytes \\
\hline Uncoded data rate, $R$ & $557 \mathrm{kbps}$ \\
\hline Time slot length, $T_{\text {slot }}$ & $1.5 \mathrm{~ms}$ \\
\hline Superframe length, $T_{s f}$ & $115.5 \mathrm{~ms}$ \\
\hline
\end{tabular}
respectively. case scenario. With respect to the objective WBAN, IEEE802.15.6 CM3 was adopted. These channel models were derived experimentally [21]. IEEE Std. 802.15.6 allows beacon shifting or channel hopping to be utilized to allow coexistence with other WBANs. This scheme was not considered in the current study, as we wished to test the robustness of our approach and the standard approach against interference from other WBANs. To do this, we investigated whether each scheme could achieve high levels of dependability under poor conditions, such as the presence of significant interference.

The main simulation parameters are listed in Table 5. The power consumption parameters for the transceivers were based on [19], [22], and [23]. In the simulations, we compared our proposed scheme with both the modes defined in the IR-UWB PHY specifications of IEEE 802.15.6 [9]: default mode with optional modulation and high QoS mode with mandatory modulation. DBPSK and DQPSK modulation were therefore utilized. In the simulations of the standard scheme, Data A was transmitted using the default mode with ordinary ARQ, whereas Data B was transmitted using the high QoS mode with hybrid ARQ. If errors were detected, the system retransmitted until the maximum number of retransmissions was reached. Then, we assumed that Data A was transmitted every superframe, while Data B was done every time slot because of each QoS. Time slot length $T_{\text {slot }}$ and superframe length $T_{s f}$ are shown in Table 5. The spreading factor $N_{s}$ was then changed based on the modulation scheme, as the data rate was fixed. For DBPSK, $N_{S}=7$, while for DQPSK, $N_{S}=15$. As our main focus was on the performance of the payload (PSDU), we assumed that the control process was completed in a time slot, and the signaling or control overhead was not considered in the simulations. Then, we mainly focused on performance of the target WBAN because our proposed scheme is closed within a WBAN and does not affect other WBANs.

Table 5. Simulation parameters. 
6.2 Numerical results Figures 10 and 11 show the RBER, and energy efficiency of the proposed scheme with each mode defined in IEEE 802.15.6 as a function of the energy per symbol to noise power spectral density $\left(E_{S} / N_{0}\right)$. The number of other WBANs was set at five. In Case 1 , the proposed scheme satisfied each QoS level under lower $E_{S} / N_{0}$ conditions, whereas the standard approach did not. The reason is that the standard scheme is not basically designed so that any QoSs can be satisfied. Hence, it can be said that the performance of each mode in the standard was simply expressed. Also, that is one of problems in the standard scheme. The RBER performance of our proposed scheme was clearly better than that of the standard scheme. For Data A, the difference between the proposed scheme and the standard scheme was approximately 8.5-10 dB. For Data B, our proposed scheme more successfully mitigated the RBER of the high QoS mode. The energy efficiency of IEEE.15.6 was 1.5-3 [information bit/ $\mu \mathrm{J}$ ] greater than that of our proposed scheme under high SNR conditions. Our proposed scheme needed retransmissions to reach the required error correction capacity, and therefore larger power consumption. However, this higher performance can be achieved under a range of SNRs. The standard scheme needed fewer transmissions under high SNR conditions as sufficient errors were removed in the first transmission. This meant that the standard did not have large power consumption due to retransmissions under high SNR conditions. However, it was unable to achieve this high performance under low or middle SNR conditions, even though the power consumption was high. The performances in Case 2 were worse than those in Case 1, especially when using the standard method. However, our proposed scheme was able to satisfy the required QoSs in the worst-case scenario under lower SNR conditions than the standard scheme. Under high SNR conditions in Case 2, the energy efficiency of our proposed scheme was better by 1.5-2.5 [information bit/ $\mu \mathrm{J}$ ] than that of IEEE 802.15.6. This was due to the error correction of the standard scheme. For our system, the energy efficiency was better for Data A than for Data B under low SNR conditions in both Cases 1 and 2. This was because the error correction capacity for Data B was insufficient, and bit errors could not be sufficiently reduced under these conditions. Table 6 shows the average difference in performance between Cases 1 and 2. The difference achieved by our proposed scheme was $4-71 \%$ smaller than that of IEEE 802.15.6. The proposed method was shown to achieve a good performance in the worst case, suggesting that it is more dependable than IEEE 802.15.6. It can be seen from Table 6 that the difference in RBER for Data B of the standard scheme was smaller than that of the proposed scheme in the DQPSK modulation. This reflects the poor RBER performance of the standard scheme under low and middle SNR conditions.

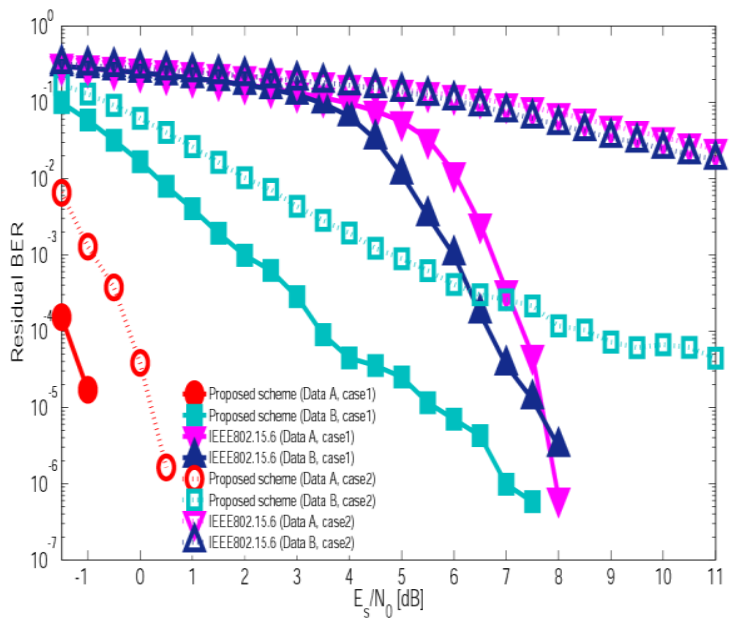

(a) DBPSK modulation

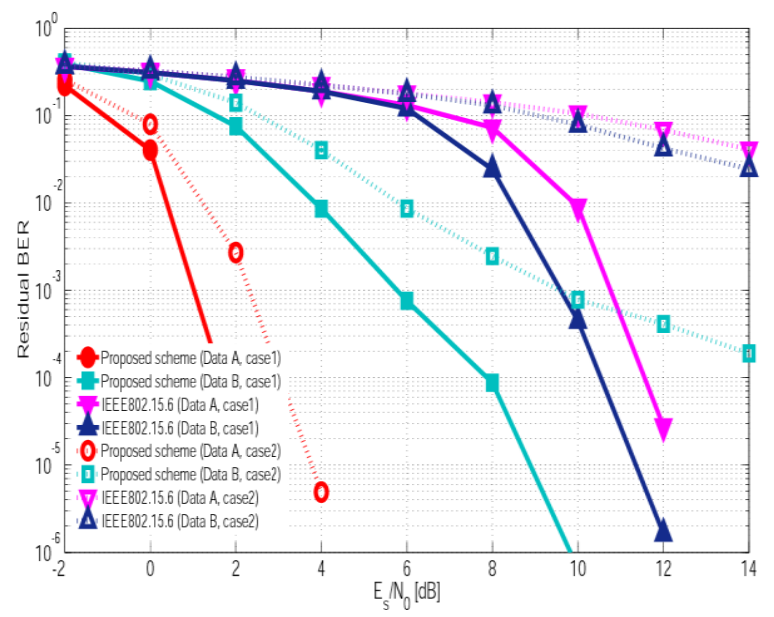

(b) DQPSK modulation

Fig. 10. RBER performance as a function of $E_{S} / N_{0}$. The number of other WBANs is five.

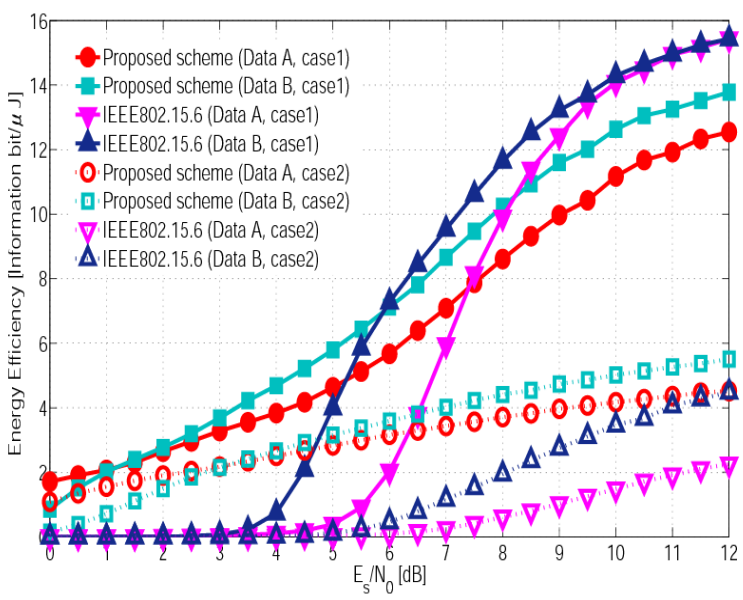

(a) DBPSK modulation 


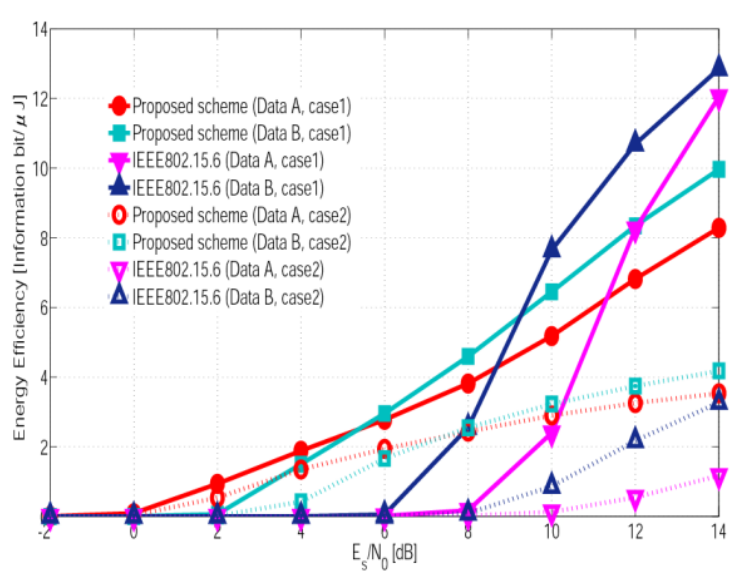

(b) DQPSK modulation

Fig. 11. Energy efficiency performance as a function of $E_{S} / N_{0}$. The number of other WBANs is five.

Table 6. Average difference in performance as a function of $E_{S} / N_{0}$ between Cases 1 and 2. The number of other WBANs is five.

\begin{tabular}{|l|c|c|c|c|}
\hline & $\begin{array}{l}\text { Data A } \\
\text { Proposal }\end{array}$ & $\begin{array}{l}\text { Data B } \\
\text { Proposal }\end{array}$ & $\begin{array}{l}\text { Data A } \\
\text { Standard }\end{array}$ & $\begin{array}{l}\text { Data B } \\
\text { Standard }\end{array}$ \\
\hline $\begin{array}{l}\log _{10} R B E R \\
\text { DBPSK }\end{array}$ & 0.7645 & 2.4947 & 2.5945 & 2.7468 \\
\hline $\begin{array}{l}\log _{10} R B E R \\
\text { DQPSK }\end{array}$ & 0.7800 & 2.1679 & 1.5146 & 1.7875 \\
\hline $\begin{array}{l}\text { Energy } \\
\text { Efficiency } \\
\text { DBPSK }\end{array}$ & 3.486 & 4.160 & 5.147 & 5.599 \\
\hline $\begin{array}{l}\text { Energy } \\
\text { Efficiency } \\
\text { DQPSK }\end{array}$ & 1.535 & 2.010 & 2.336 & 3.044 \\
\hline
\end{tabular}

Figures 12 and 13 show the energy efficiency, and RBER performance of the proposed scheme at each mode defined in IEEE802.15.6 as a function of the number of other WBANs. $E_{S} / N_{0}$ was set to $5 \mathrm{~dB}$ in the DBPSK modulation and $8 \mathrm{~dB}$ in the DQPSK modulation, respectively. The results mirrored those presented in Figures 9 and 10. In Figure 11 (a), the RBER of Data A is not plotted because all errors were removed by the large error correcting capacity. As the number of other WBANs increased, the performance deteriorated. However, the proposed scheme satisfied the QoSs for two data sets. In contrast, the standard performed very poorly, especially in Case 2. This suggested that the standard scheme was not robust against strong interference from other WBANs. In Case 1, the energy efficiency performance of the high QoS mode was inferior to those for Data A of the proposed scheme, with a maximum difference in energy efficiency of 2 [information bit/ $\mu \mathrm{J}]$. The standard scheme had a poor RBER performance under small interference conditions in both modulation cases, whereas the RBER performance of our scheme under the same conditions was good. The superior performance of our scheme reflects the analysis in [12]-[14]. In these simulations, parameters such as $E_{S} / N_{0}$ were taken from these references. Table 7 summarizes the difference in performance between Cases 1 and 2. The difference for Data A in IEEE 802.15 .6 for both modulation cases was very small because of the very poor performance in both Cases 1 and 2. Then, the difference for Data B of IEEE 802.15.6 was smaller than that of our system in the DQPSK modulation, because of its poor performance in the worst case.

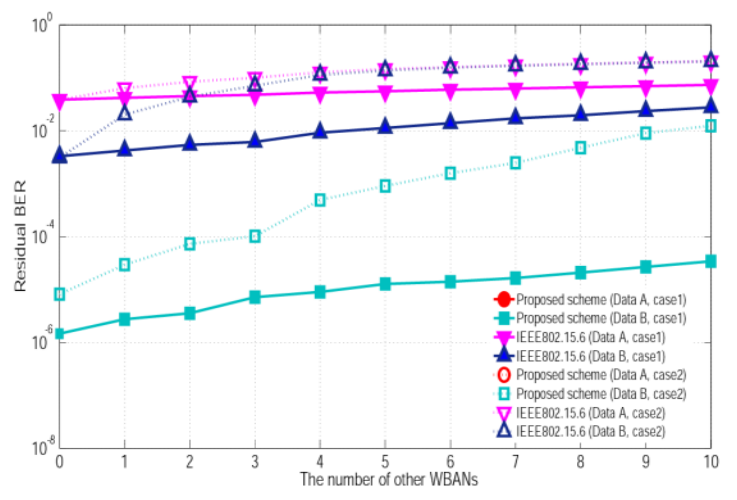

(a) DBPSK modulation. $E_{S} / N_{0}=5 \mathrm{~dB}$.

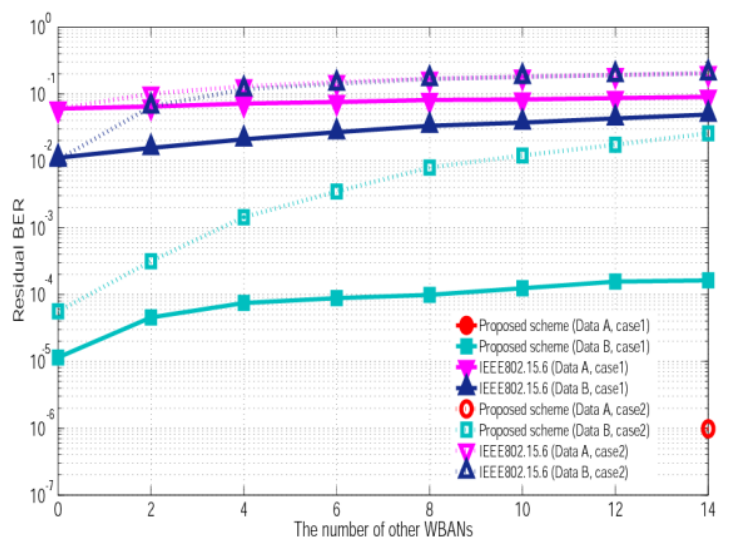

(b) DQPSK modulation. $E_{S} / N_{0}=8 \mathrm{~dB}$.

Fig. 12. RBER performance as a function of the number of other WBANs.

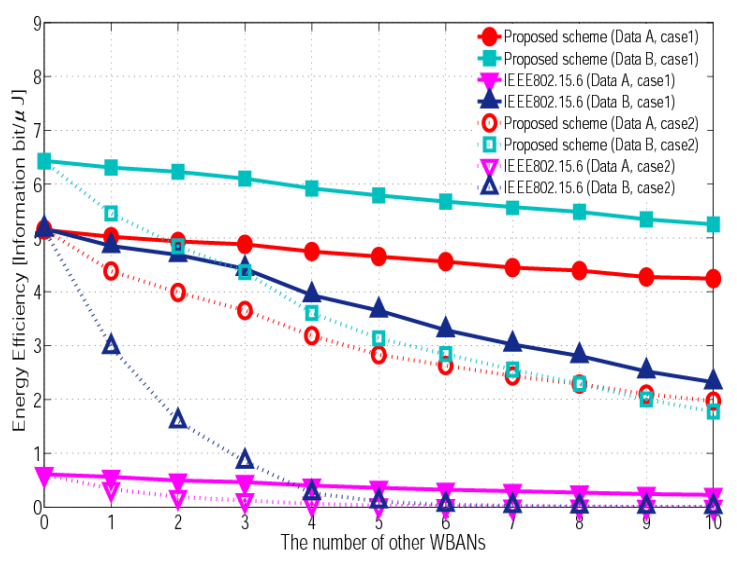

(a) DBPSK modulation. $E_{S} / N_{0}=5 \mathrm{~dB}$. 


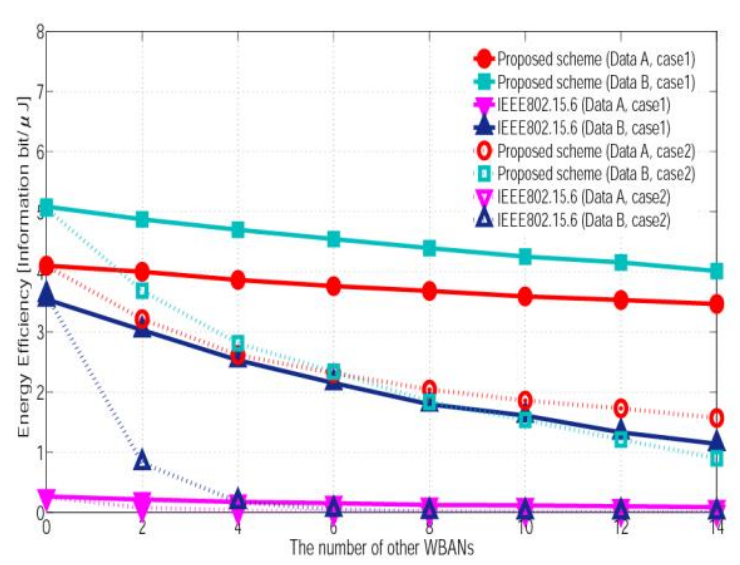

(b) DQPSK modulation. $E_{S} / N_{0}=8 \mathrm{~dB}$.

Fig. 13. Energy efficiency performance as a function of the number of other WBANs.

Table 7. Average difference in performance as a function of the number of other WBANs between Cases 1 and 2. $E_{S} / N_{0}=5 \mathrm{~dB}$ (DBPSK) and $8 \mathrm{~dB}$ (DQPSK).

\begin{tabular}{|l|c|c|c|c|}
\hline & $\begin{array}{l}\text { Data A } \\
\text { Proposal }\end{array}$ & $\begin{array}{l}\text { Data B } \\
\text { Proposal }\end{array}$ & $\begin{array}{l}\text { Data A } \\
\text { Standard }\end{array}$ & $\begin{array}{l}\text { Data B } \\
\text { Standard }\end{array}$ \\
\hline $\begin{array}{l}\log _{10} R B E R \\
\text { DBPSK }\end{array}$ & 0 & 1.7269 & 0.3428 & 0.8721 \\
\hline $\begin{array}{l}\log _{10} R B E R \\
\text { DQPSK }\end{array}$ & 0.4989 & 1.5683 & 0.2628 & 0.5891 \\
\hline $\begin{array}{l}\text { Energy } \\
\text { Efficiency } \\
\text { DBPSK }\end{array}$ & 1.517 & 2.256 & 0.2595 & 2.695 \\
\hline $\begin{array}{l}\text { Energy } \\
\text { Efficiency } \\
\text { DQPSK }\end{array}$ & 1.320 & 2.080 & 0.106 & 1.556 \\
\hline
\end{tabular}

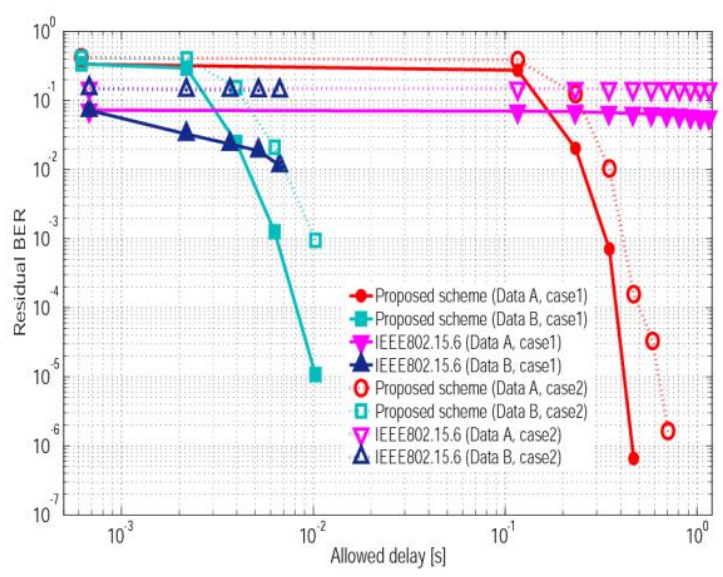

(a) $E_{s} / N_{0}=5 \mathrm{~dB}$.

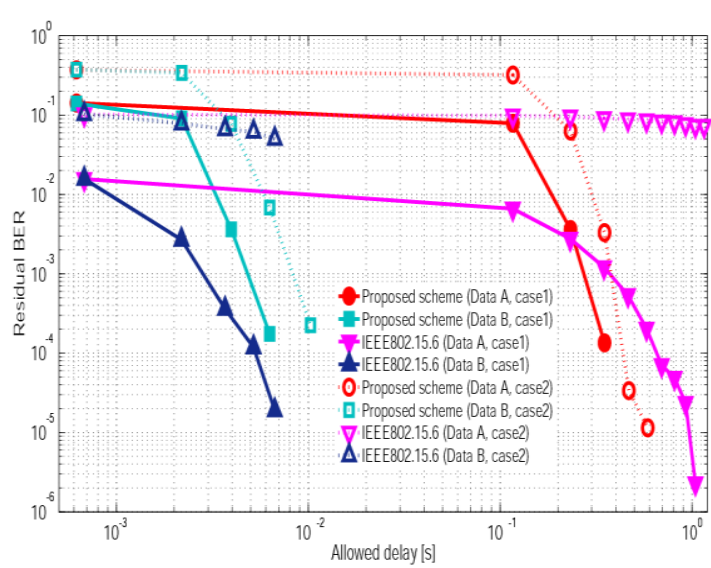

(b) $E_{S} / N_{0}=8 \mathrm{~dB}$.

Fig. 14. RBER performance as a function of an allowed delay. The number of other WBANs is five and the modulation is DBPSK

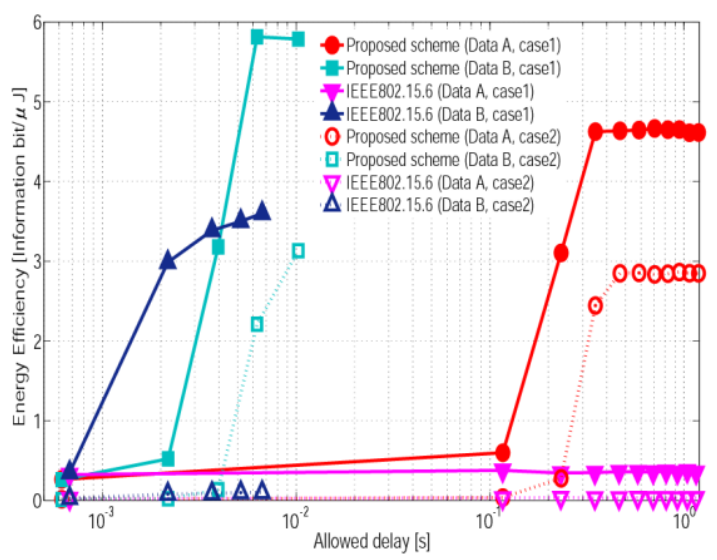

(a) $E_{S} / N_{0}=5 \mathrm{~dB}$.

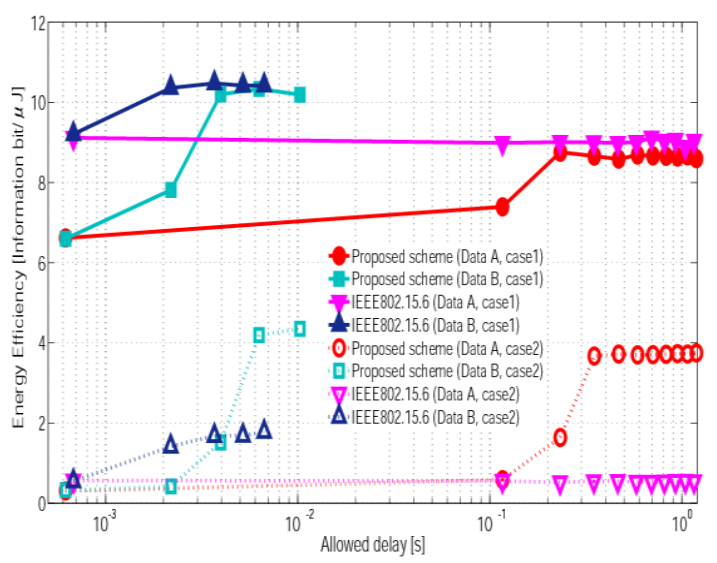

(b) $E_{S} / N_{0}=8 \mathrm{~dB}$

Fig. 15. Energy efficiency performance as a function of an allowed delay. The number of other WBANs is five and the modulation is DBPSK 
Table 8. Average difference in performance as a function of an allowed delay between Cases 1 and 2 (the number of other WBANs is five and the modulation is DBPSK).

\begin{tabular}{|l|c|c|c|c|}
\hline & $\begin{array}{l}\text { Data A } \\
\text { Proposal }\end{array}$ & $\begin{array}{l}\text { Data B } \\
\text { Proposal }\end{array}$ & $\begin{array}{l}\text { Data A } \\
\text { Standard }\end{array}$ & $\begin{array}{l}\text { Data B } \\
\text { Standard }\end{array}$ \\
\hline $\begin{array}{l}\log _{10} R B E R \\
E_{S} / N_{0}=5 \mathrm{~dB}\end{array}$ & 1.3006 & 0.8380 & 0.3643 & 0.7374 \\
\hline $\begin{array}{l}\log _{10} R B E R \\
E_{S} / N_{0}=8 \mathrm{~dB}\end{array}$ & 1.2948 & 2.0552 & 3.0389 & 2.1378 \\
\hline $\begin{array}{l}\text { Energy } \\
\text { Efficiency } \\
E_{S} / N_{0}=5 \mathrm{~dB}\end{array}$ & 1.668 & 2.010 & 0.3237 & 2.685 \\
\hline $\begin{array}{l}\text { Energy } \\
\text { Efficiency } \\
E_{S} / N_{0}=8 \mathrm{~dB}\end{array}$ & 5.426 & 6.868 & 8.480 & 8.757 \\
\hline
\end{tabular}

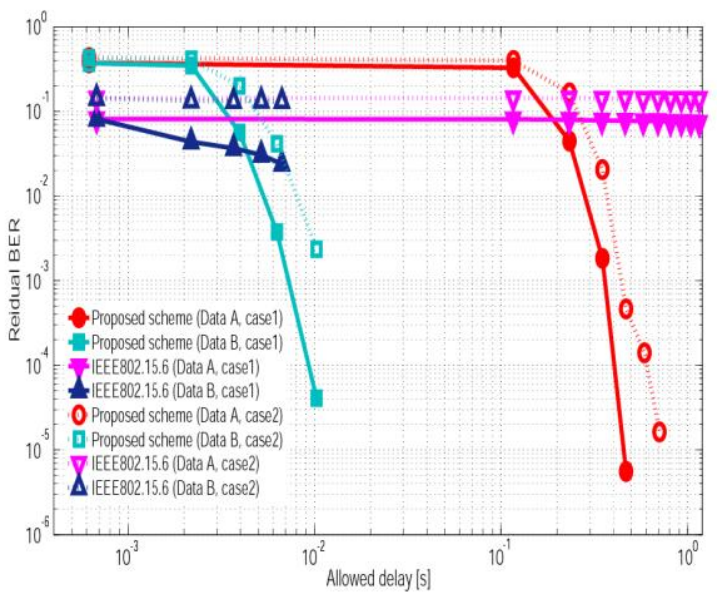

(a) $E_{S} / N_{0}=8 \mathrm{~dB}$

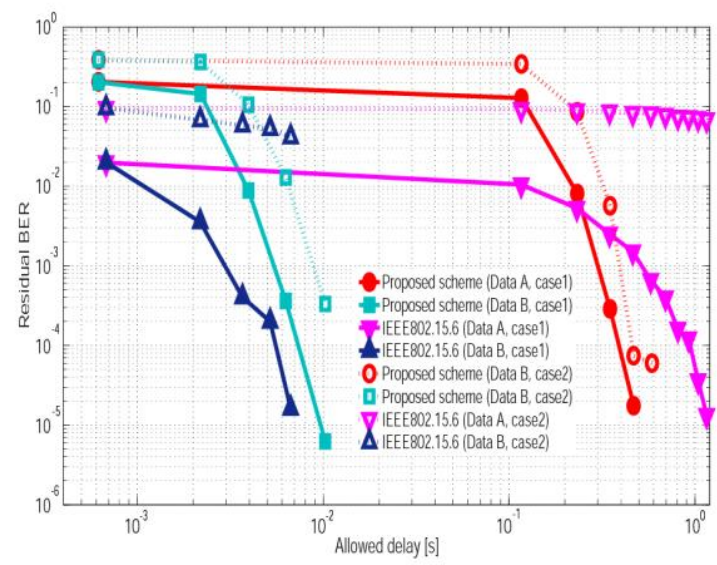

(b) $E_{S} / N_{0}=12 \mathrm{~dB}$

Fig. 16. RBER performance as a function of an allowed delay. The number of other WBANs is five and the modulation is DQPSK

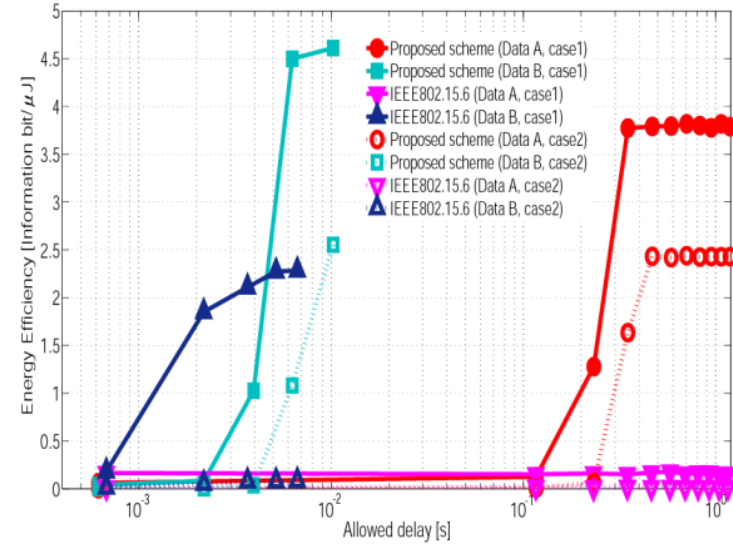

(a) $E_{s} / N_{0}=8 \mathrm{~dB}$

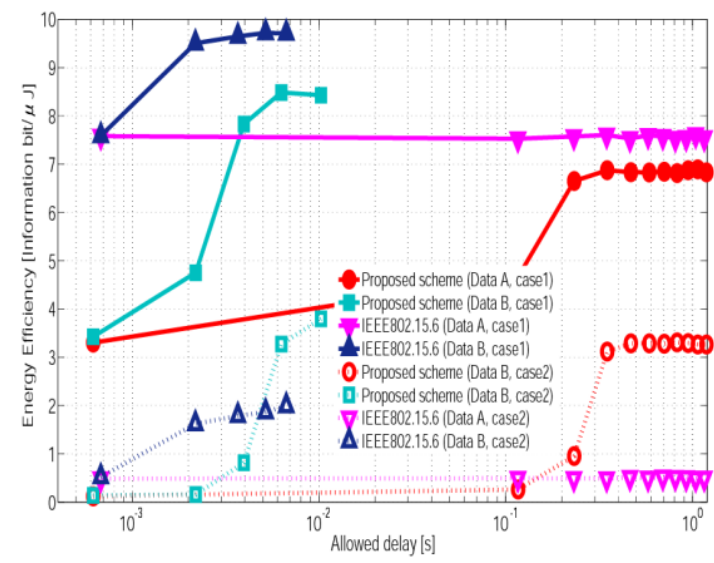

(b) $E_{S} / N_{0}=12 \mathrm{~dB}$.

Fig. 17. Energy efficiency performance as a function of an allowed delay. The number of other WBANs is five and the modulation is DQPSK

Table 9. Average difference in performance as a function of an allowed delay between Cases 1 and 2 (the number of other WBANs is five and the modulation is DQPSK).

\begin{tabular}{|l|l|l|l|l|}
\hline & $\begin{array}{l}\text { Data A } \\
\text { Proposal }\end{array}$ & $\begin{array}{l}\text { Data B } \\
\text { Proposal }\end{array}$ & $\begin{array}{l}\text { Data A } \\
\text { Standard }\end{array}$ & $\begin{array}{l}\text { Data B } \\
\text { Standard }\end{array}$ \\
\hline $\begin{array}{l}\log _{10} R B E R \\
E_{S} / N_{0}=8 \mathrm{~dB}\end{array}$ & 1.3684 & 0.6987 & 0.2646 & 0.5324 \\
\hline $\begin{array}{l}\log _{10} R B E R \\
E_{S} / N_{0}=12 \mathrm{~dB}\end{array}$ & 0.8575 & 1.0078 & 2.0877 & 1.9898 \\
\hline $\begin{array}{l}\text { Energy } \\
\text { Efficiency } \\
E_{S} / N_{0}=8 \mathrm{~dB}\end{array}$ & 1.193 & 1.316 & 0.1465 & 1.677 \\
\hline $\begin{array}{l}\text { Energy } \\
\text { Efficiency } \\
E_{S} / N_{0}=12 \mathrm{~dB}\end{array}$ & 3.817 & 4.949 & 7.067 & 7.677 \\
\hline
\end{tabular}




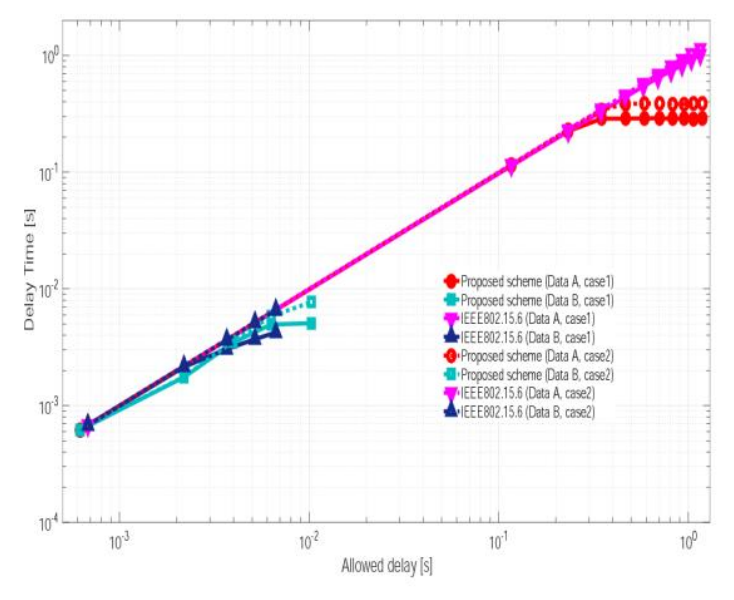

(a) $E_{S} / N_{0}=5 \mathrm{~dB}$.

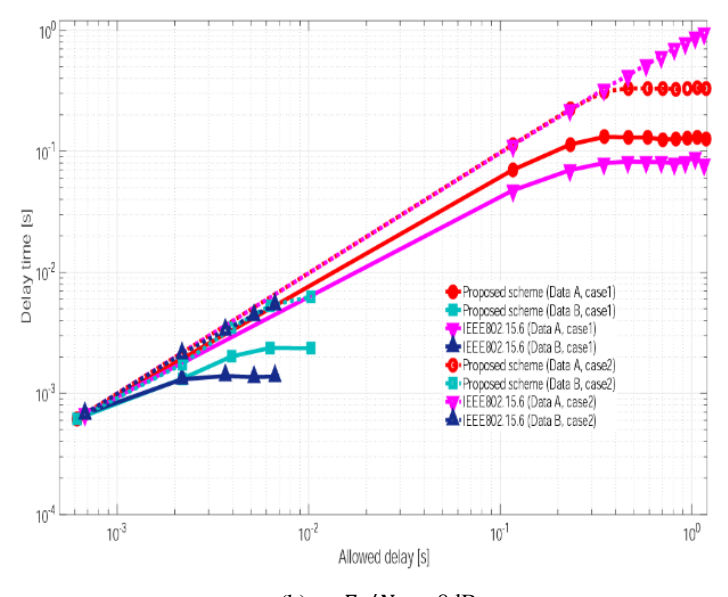

(b) $E_{S} / N_{0}=8 \mathrm{~dB}$.

Fig. 18. Delay time as a function of an allowed delay. The number of other WBANs is five and the modulation is DBPSK

Table 10. Average difference in delay time as a function of an allowed delay between Cases 1 and 2 (the number of other WBANs is five and the modulation is DBPSK).

\begin{tabular}{|c|c|c|c|c|}
\hline & $\begin{array}{l}\text { Data A } \\
\text { Proposal }\end{array}$ & $\begin{array}{l}\text { Data B } \\
\text { Proposal }\end{array}$ & $\begin{array}{l}\text { Data A } \\
\text { Standard }\end{array}$ & $\begin{array}{l}\text { Data B } \\
\text { Standard }\end{array}$ \\
\hline $\begin{array}{c}E_{S} / N_{0}=5 \mathrm{~dB} \\
{[\mathrm{~ms}]}\end{array}$ & 67.9 & 0.326 & 47.0 & 0.404 \\
\hline $\begin{array}{c}E_{S} / N_{0}=8 \mathrm{~dB} \\
{[\mathrm{~ms}]}\end{array}$ & 159 & 0.794 & 432 & 0.893 \\
\hline
\end{tabular}

Figures 14 and 15 show the performance as a function of allowed delay with $E_{S} / N_{0}=5 \mathrm{~dB}$ and $E_{S} / N_{0}=8 \mathrm{~dB}$ using DBPSK modulation. The allowed delay affects the number of retransmissions that can be performed within the latency requirements for the particular data type. Those figures compare performances between under low and high SNR conditions. So, we assumed that the number of other WBANs was five because an influence of interference from other WBANs was not too large and not too small in that case from Fig. 12 and 13. It can be seen that as the allowed delay became longer, the performance of the proposed scheme improved. This was especially true under poor channel conditions such as those shown in Figures 14 and 15, because the error correction for each retransmission was increased. These delays were acceptable for the target applications. In contrast, the performance of the standard method did not improve at low SNRs, even when the allowed delay was increased. This is because error correction did not increase with retransmission. In Case 1, the maximum energy efficiency of our proposed scheme was 2.5 [Information bit $/ \mu \mathrm{J}$ ] higher than that of the standard scheme for Data $\mathrm{B}$, and approximately 4.5 [Information $\mathrm{bit} / \mu \mathrm{J}$ ] greater than the standard for Data A. In Case 2, the maximum energy efficiency was approximately 3 [Information bit/ $\mu \mathrm{J}$ ] higher than that of IEEE 802.15.6 for both Data A and Data B. Under high SNR conditions, the standard had a better performance at smaller allowed delay times than the proposed scheme in the general case. This was because the error correction of the standard was sufficient to remove errors at the first transmission. The maximum energy efficiency of the standard was 0.2 [Information $\mathrm{bit} / \mu \mathrm{J}$ ] greater than that of the proposed system for both Data A and Data B. However, this difference is very small. In contrast, the performance of our proposed scheme was superior to that of IEEE 802.15.6 in the worst case, because the error correction of the standard scheme was insufficient in this case and the standard scheme was unable to increase the error correction at each retransmission. The maximum energy efficiency of our proposed method was 3.5 [Information bit/ $\mu \mathrm{J}]$ larger than that of IEEE 802.15.6 for Data A. Tables 8 shows the difference in performance as a function of the allowed delay between Cases 1 and 2. As can be seen from Table 8, the standard scheme had a smaller difference for Data A, since the performance was poor in both cases. In terms of energy efficiency, our proposed scheme was able to reduce the difference more successfully than IEEE 802.15 .6 because of its superior error correction. Table 8 also shows a larger difference under the high SNR condition. Especially, the standard scheme had larger difference because it was not robust against the strong interference in the worst case scenario. Our scheme achieved a difference 4-57\% smaller than that of the standard scheme.

Figures 16-17 and Table 9 show the results as a function of the allowed delay with $E_{S} / N_{0}=8$ and $12 \mathrm{~dB}$ using DQPSK modulation. The results mirrored those for DBPSK modulation.

Finally, Figure 18 shows delay time as a function of an allowed delay with $E_{S} / N_{0}=5 \mathrm{~dB}$ and $E_{S} / N_{0}=8 \mathrm{~dB}$ using DBPSK modulation and Table 10 shows the difference in delay time as a function of an allowed delay between both cases. These figures a few examples of the delay time for the typical retransmission cases. Then, we show them in order to understand the relationship between the allowed delay and the number of retransmission. The delay time in case of $i$ times transmissions was calculated as follows:

$$
T_{d e l, i}=\frac{L_{\text {packet }, i}}{R}+(i-1) T_{\text {int }} .
$$

Here, $T_{\text {int }}$ is a transmission interval. Hence, it was assumed that $T_{\text {int }}=T_{s f}$ in case of Data A, while $T_{\text {int }}=T_{\text {slot }}$ in case of Data B. It was shown that the delay time was shorter than the allowed delay from those figures. This is because all errors were corrected before the maximum number of retransmissions was reached. Under poor channel conditions, differences of the delay time between both cases were small in both schemes. On the other hand, those 
differences were larger from results under high SNR conditions. However, those of the proposed scheme was smaller than the standard scheme. Hence, it can be said that our proposed scheme was able to guarantee the delay time in both cases. By the way, the result in a QPSK case was not shown as an example because that was almost the same as the result for DBPSK modulation.

These results demonstrate that our proposed scheme achieved high performance in a multiple WBAN environment. It can be further observed that IEEE 802.15.6 failed to achieve a satisfactory performance under the worst-case scenario, while our proposed scheme was able to do so under both scenarios

\section{Conclusions and future work}

In this research, we proposed an error control method using decomposable error correcting codes. We used this to optimize a range of QoS standards. Computer simulations were conducted to evaluate the proposed scheme against performance measures such as RBER and energy efficiency and to compare the results with those from an error control scheme defined in IEEE 802.15.6, using both DBPSK and DQPSK modulation. The results demonstrated that the proposed scheme was able to satisfy the required QoS for each data type, and that the QoS control was more flexible than that of the standard in both the general and worst-case scenarios. The proposed scheme had poorer energy efficiency than IEEE 802.15.6 under high SNR conditions in the general case scenario. However, the IEEE 802.15.6 solution was unable to achieve the required performance under the worst-case scenario. It can be concluded that the proposed error control coding scheme enables QoS control while maintaining high, or satisfactory, energy efficiency under the different scenarios.

In future work, we will address the following issues. A theoretical analysis of the channel models was not attempted in this paper because the models were experimentally derived. We will investigate the optimal parameters for the channel model using a theoretical analysis. A cross-layer optimization of our error control scheme combining schedule based and random access MAC protocols will also be conducted. Then, we should consider effective interference countermeasure in a multiple WBANs environment. Finally, the control overhead will also be considered.

\section{References}

(1) Chen, M., Gonzalez, S., Vasilakos, A., Cao, H. and Leung, V. C. M.: "Body Area Networks: A Survey. Mobile Networks and Applications", Springer, Vol.16, No.2, pp.171-193 (2010)

(2) Acampora, G., Cook, D.J. Rashidi and P. Vasilakos, A.V.: "A Survey on Ambient Intelligence in Healthcare", Proceedings of the IEEE, Vol.101, No.12,pp. 2470-2494 (2013)

(3) Hayajneh, T., Almashaqbeh, G., Ullah, S. and Vasilakos, A.V.: "A survey of wireless technologies coexistence in WBAN: analysis and open research issues", Wireless Networks, Springer, Vol.20, No.8, pp.2165-2199 (2014)

(4) Fortino, G., Fatta, G. D., Pathan, M. and Vasilakos, A.V.: "Cloud-assisted body area networks: state-of-the-art and future challenges", Wireless Networks, Springer, Vol.20, No.7, pp.1925-1938 (2014)

(5) Cao, H., Leung, V,. Chow, C. and Chan, H.: "Enabling technologies for wireless body area networks: A survey and outlook", Communications Magazine, IEEE, Vol.47, No.12, pp.84-93 (2009)

(6) Viswanathan, H., Chen, B. and Pompili, D.: "Research challenges in computation, communication, and context awareness for ubiquitous healthcare", Communications Magazine, IEEE, Vol.50, No.5, pp.92-99 (2012)

(7) Bachmann, C., Ashouei, M., Pop, V., Vidojkovic, M., Groot, H.D. and Gyselinckx, B.: "Low-power wireless sensor nodes for ubiquitous long-term biomedical signal monitoring", Communications Magazine, IEEE, Vol.50, No.1, pp.20-27 (2012)
(8) Suzuki, T., Tanaka, H., Minami, S., Yamada, H. and Miyata, T.: "Wearable Wireless Vital Monitoring Technology for Smart Health Care", Proceedings of 7th International Symposium on Medical Information and Communication Technology (ISMICT), March 2013; Tokyo, Japan (2013)

(9) IEEE Standard for Information technology - Telecommunications and information exchange between systems - Local and metropolitan area networks- Specific requirements, "Part 15.6: Wireless Medium Access Control (MAC) and Physical Layer (PHY) Specifications for Wireless Personal Area Networks (WPANs) used in or 12 around a body", IEEE (2011)

(10) ETSI TC Smart BAN, "Smart Body Area Network (SmartBAN); Low Complexity Medium Access Control (MAC) for SmartBAN", TS 103325 V1.1.1 (2015)

(11) Takabayashi, K., Tanaka, H., Sugimoto, C. and Kohno, R.: "Multiplexing and Error Control Scheme for Body Area Network employing IEEE 802.15.6”, Transactions on Communications, IEICE, Vol.E97-B, No.03, pp.564-570 (2014)

(12) Takabayashi, K., Tanaka, H., Sugimoto, C. and Kohno, R.: "Performance Analysis of Multiplexing and Error Control Scheme for Body Area Networks" EURASIP Journal on Wireless Communications and Networking, Vol.2016, No.70 (2016)

(13) Takabayashi, K., Tanaka, H., Sugimoto, C. and Kohno, R.: "Error Control Scheme Using Decomposable Codes for Various QoS in Multiple WBAN Environment", Proceedings of 9th Int. Symp. Medical Information and Communication Technology (ISMICT 2015), March 2015; Kamakura, Japan (2015)

(14) Takabayashi, K., Karvonen, H., Paso, T., Tanaka, H., Sugimoto, C. and Kohno, R.: "Energy Efficiency Evaluation of ECC Scheme Utilizing Decomposable Codes in IEEE Std 802.15.6 Based WBANs", Proceedings of 10th International Conference on Body Area Networks (BodyNets 2015), September 2015; Sydney, Australia (2015)

(15) P11073-00101/D02J. Health informatics - Point-of-care medical device communication - Technical report - "Guidelines for the use of RF wireless technology", IEEE (2007)

(16) Obinata, Y., Yamasue, K., Pham, T. H., Nemoto, A., Sugimoto, C. and Kohno, R.: "Application of High-band UWB Body Area Network to Medical Vital Sensing in Hospital", Proceedings of The Second Ultra Wideband for Body Area Networking Workshop (UWBAN-2013), October 2013; Boston, MA, US (2013)

(17) Weldon, E., Jr. "An Improved Selective-Repeat ARQ Strategy", Transactions on Communications, IEEE, Vol.30, No.3, pp.480-486 (1982)

(18) Takabayashi, K., Tanaka, H., Sugimoto, C. and Kohno, R.: "Effective Error Control Scheme with Channel State Information for WBAN", Proceedings of 8th Int. Symp. Medical Information and Communication Technology (ISMICT 2014), April 2014; Florence, Italy (2014)

(19) Karvonen, H., Iinatti, J., Hämäläinen, M., "A cross-layer energy efficiency optimization model for WBAN using IR-UWB transceivers", Springer Telecommunication Systems, Vol.58, No.2, pp.165-177 (2015)

(20) Abughalieh, N., Steenhaut, K., Now'e, A., Anpalagan, A.: "Turbo codes for multihop wireless sensor networks with decode-and-forward mechanism", EURASIP Journal on Wireless Communications and Networking, Vol.204, No.1 (2014)

(21) Yazdandoost , K. Y., Sayrafian-Pour, K.: "Channel Model for Body Area Network (BAN)" IEEE P802.15Working Group for Wireless Personal Area Networks( WPANs), IEEE P802.15-08-0780-10-0006.14 (2009)

(22) Ryckaert, J., Desset, C., Fort, A., Badaroglu, M., et al.: "Ultra-Wide-Band Transmitter for Low-Power Wireless Body Area Networks: Design and Evaluation”, IEEE Trans. Circuits Syst. I, Reg. Papers, Vol.52, No.12, pp.2515-2525 (2005)

(23) Zou, Z., Mendoza, D. S., Wang, P., Zhou, Q., et al.: "A Low-Power and Flexible Energy Detection IR-UWB Receiver for RFID and Wireless Sensor Networks", IEEE Trans. Circuits Syst. I, Reg. Papers, Vol.58, No.7, pp.1470-1482 (2011). 
Kento Takabayashi (Non-Member) Kento Takabayashi received the

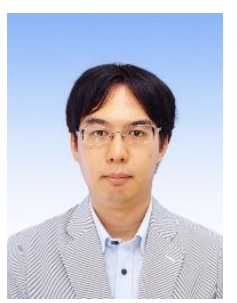
Master's degree in Electrical and Computer Engineering from Yokohama National University, Yokohama, Japan, in 2014. He is currently working toward the Ph.D. degree in Physics, Electrical and Computer Engineering at Yokohama National University. His research interests in MICT such as Wireless Body Area Network and coding theory in wireless communications.

Heikki Karvonen

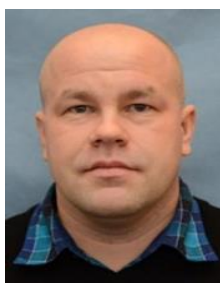

(Non-Member) Heikki Karvonen received Master of Science degree (electrical engineering) in 2004 and Doctor of Technology (telecommunications) degree in 2015 from the University of Oulu, Finland. Currently he works as a Post-doctoral researcher at the Centre for Wireless Communications (CWC), University of Oulu, Finland. He has experience of working as a research scientist and/or project manager in several national and international research projects carried out at the CWC during the years 2003-2016. In his research the main focus has been on the energy efficiency improvement of wireless sensor and body area networks (WSN \& WBAN) by using (PHY / MAC) protocol's cross-layer optimization. He has also experience of design of architecture for Internet of Things (IoT) and 4G/5G telecommunication network technologies development.

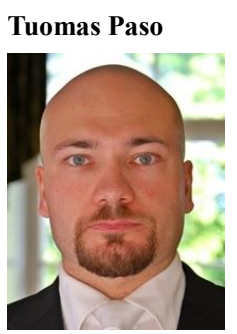

(Non-Member) Tuomas Paso received his M.Sc. degree in telecommunications engineering from University of Oulu, Finland, in March 2010. Currently he is working as a research scientist and as a doctoral student in Centre for Wireless Communications, University of Oulu. His research interests include MAC protocols and PHY/MAC/NET cross-layers techniques in MANETs and wireless sensor networks, such as WBANs and WPANs. The main focus areas of his research are healthcare/medical ICT and security/defence. Since 2013 he has been a member and an active contributor of ETSI TC SmartBAN, which is a technical committee established to define a European body area networks standard.

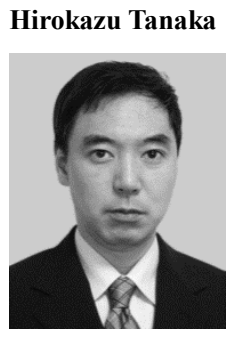

(Non-Member) Hirokazu Tanaka received his B.E. and $\mathrm{Ph} . \mathrm{D}$. degrees in Communications Engineering from Osaka University, Suita, Osaka in 1989 and 2001, respectively. He joined Toshiba Corporation as a researcher in 1989. Since 2015, he has been a professor of Hiroshima City University. From 2007 to 2009 , he was a visiting researcher at Hokkaido University and served as a visiting associate professor of Hokkaido University from 2007 to 2008. Since 2013, he has been a visiting associate professor of Yokohama National University. Since 1997, Dr. Tanaka has been involved with international standardization activities in the field of mobile multimedia systems in ITUT, 3GPP, 3GPP2 and Bluetooth. He actively contributed to defining technical specifications of video telephony systems, multimedia streaming systems, multimedia messaging systems, etc. for 3rd-generation wireless communication systems. Since 2013, he has been serving as the vice chair of TC Smart BAN in European Telecommunications Standards Institute (ETSI). He received the ITU-AJ Award from the ITU Association of Japan in 1999. His research interests include theory and applications of modulation,

error control coding, access control and audio/video coding, with emphasis on applications to mobile multimedia communications, satellite communications, broadcasting and dependable wireless communications. Dr. Tanaka is a senior member of IEEE.

Chika Sugimoto

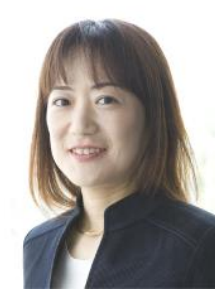

Ryuji Kohno

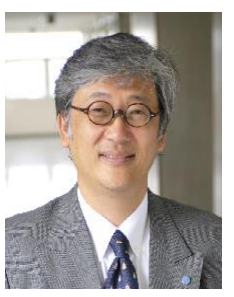

(Non-Member) Chika Sugimoto received the B.S. degree in Engineering, and the M.S. and the Ph.D. degrees in Environment from The University of Tokyo, respectively. During 2006-2010, she was an assistant professor of Graduate school of Frontier Sciences, The University of Tokyo. Since 2010 she is an associate professor of Graduate School of Engineering, National Yokohama University. She is a member of IEEE.

(Member) Ryuji Kohno is IEICE and IEEE fellows. He received the Ph.D. degree in Dept.EE from the Univ. of Tokyo in 1984. Since 1998 he has been a Professor in YNU. During 1984-1985 he was a Visiting Scientist in Dept.EE, Univ. of Toronto. Since 2007, he is also a Finnish Distinguished Professor (FiDiPro) in Univ. of Oulu, Finland. The meanwhile, he was also a director of SONY CSL/ATL during 1998-2002 and was a director of the UWB Tech. Inst. and a program coordinator of Medical ICT Inst. of National Institute of Information and Communications Technology (NICT) during 2002-2011. Currently he is the CEO of the University of Oulu Research Institute Japan

- CWC-Nippon Inc. Ltd. since March 2012. He was a principal leader of MEXT 21st century and Global COE programs during 2002-2007 and 20082013, respectively. Since 2003, he is a director of Medical ICT Center in YNU. He is an associate member of the Science Council of Japan since 2006. Prof. Kohno was elected to be a BoG member of the IEEE Information Theory Society three times on 2000, 2002, and 2006. He was awarded IEICE Greatest Contribution Award and NTT DoCoMo Mobile Science Award in 1999 and 2002, respectively. 